\title{
Anesthetic considerations in Demons-Meigs' syndrome: a case report
}

\author{
Salaheddine Fjouji, Mustapha Bensghir ${ }^{*}$, Charki Haimeur and Hicham Azendour
}

\begin{abstract}
Introduction: Demons-Meigs' syndrome is characterized by the presence of a benign ovarian tumor associated with ascites and a right-sided hydrothorax. Its pathophysiology remains unclear. Anesthesia of this syndrome is a real challenge. Respiratory, hemodynamic, metabolic problems and abdominal hypertension are the main anesthetic risks.

Case presentation: A 52-year-old African woman with Demons-Meigs' syndrome was admitted for elective surgery under general anesthesia. An abdominal computed tomography scan showed a tumor mass, with tissue and cystic components associated with abundant ascites and a right pleural effusion of medium abundance. In the operating room after standard monitoring, a crash induction was performed. Just after, her saturation level decreased requiring the use of an alveolar recruitment maneuver followed by the application of positive end-expiratory pressure. Vasoconstrictor and vascular filling were used to correct the hypotension that occurred. Airway pressures remained at $35 \mathrm{~cm} \mathrm{H} 2 \mathrm{O}$. Maintenance of a slightly proclive position and opening of the abdomen with the progressive removal of 3200ml ascitic fluid allowed a lower thoracic pressure (airway pressures $=24 \mathrm{~cm} \mathrm{H2O}$ ). Her postoperative course was unremarkable. Clinical evolution after five months was marked by a complete recovery of our patient and no recurrence of effusion or ascites.
\end{abstract}

Conclusions: Demons-Meigs' syndrome is a benign disease with a good prognosis. Respiratory and hemodynamic problems and abdominal hypertension are the main anesthetic risks of this syndrome. Good management of these risks is necessary to preserve the prognosis.

Keywords: Demons-Meigs' syndrome, Anesthesia, Ascites, Pleural effusion, Abdominal hypertension

\section{Introduction}

Demons-Meigs' syndrome is characterized by the presence of a benign ovarian tumor associated with ascites and a right-sided hydrothorax [1,2].

It is rarely seen and its pathophysiology remains unclear [3,4]. Differential diagnosis with ovarian neoplasia should be discussed before surgery. The pleural effusion and ascites resolve spontaneously and permanently after removal of the tumor [5].

Anesthesia of this syndrome is a real challenge. Respiratory, hemodynamic and metabolic problems and abdominal hypertension are the main anesthetic risks [6-8]. Management of these risks is a perioperative priority. The authors present a clinical case involving the anesthetic management of this syndrome and a review of the literature.

\footnotetext{
* Correspondence: mustaphabens_15rea@hotmail.com

Department of Anaesthesiology, Military Hospital Med V Rabat, University of Med V Souissi, Avenue des Nations Unies, Rabat 10000, Morocco
}

\section{Case presentation}

A 52-year-old African woman with primary infertility who had been postmenopausal for eight years presented with abdominal heaviness of nine months' duration recently associated with postprandial vomiting. No change of her general state was noted. There were no signs of compression of the pelvic organs. Abdominal ultrasonography showed a huge abdominal mass and an effusion of average abundance. An abdominal computed tomography (CT) scan (Figure 1) showed a tumor mass, tissue and a cystic component measuring $189 \times 133 \times 291 \mathrm{~mm}$ displacing the bowel loops up and out, and the bladder and uterus down, associated by abundant ascites and a right pleural effusion of medium abundance. The level of carbohydrate antigen 125 (CA-125) was $133 \mathrm{U} / \mathrm{ml}$. Surgical exploration was indicated. A preoperative examination noted NYHA (New York Heart Association) class II dyspnea, and obesity with a body mass index (BMI) of $38.6 \mathrm{~kg} / \mathrm{m}^{2}$. An examination of her upper airway noted a Mallampati grade II. Auscultation 


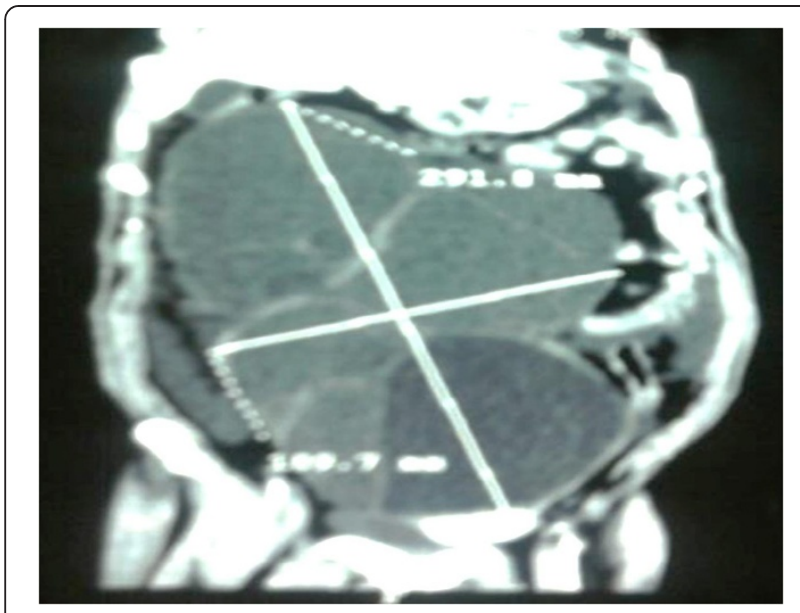

Figure 1 Computed tomography scan showing a huge tumor with tissue and cystic components.

found silence at the base of her right lung. Her abdomen was distended. Her pulse oximetry indicated $97 \%$. Her electrocardiogram was normal. A preoperative chest $\mathrm{X}$-ray showed effusion syndrome of medium amount. Echocardiography showed good cardiac function with an ejection fraction of $68 \%$. There were no electrolytic disorders: potassium at $4.2 \mathrm{mmol} / \mathrm{l}$, fasting plasma glucose at $1.05 \mathrm{~g} / \mathrm{l}$. Her renal function was not impaired, and hemostasis laboratory tests were normal. Her preoperative hemoglobin was $12.2 \mathrm{~g} / \mathrm{dl}$. Our patient was premedicated with $50 \mathrm{mg}$ hydroxizine the day before surgery and $50 \mathrm{mg}$ on the morning of the operation. In the operating room, standard monitoring (noninvasive blood pressure, scope, arterial oxygen saturation) was performed. Venous access was secured by two $16 \mathrm{G}$ catheters and $2 \mathrm{~g}$ cefazolin was administered. After 10 minutes of preoxygenation in a twenty-degree reverse-Trendelenburg position, and a vascular filling of $1000 \mathrm{ml}$ of crystalloid, the crash induction was administered using 100mg suxamethonium, 500mg thiopental and the Sellick's maneuver. Oral tracheal intubation was successful at the first attempt by a tube $7 \mathrm{~mm}$ in diameter and our patient was connected to the anesthesia machine and ventilated with a tidal volume of $480 \mathrm{ml}$, respiratory rate of 14 cycles/minute. Just after connecting our patient to the ventilator, her saturation level decreased to $96 \%$. To treat and prevent a secondary drop in saturation, an alveolar recruitment maneuver was decided upon. This maneuver was performed by the application of continuous positive airway pressure $(40 \mathrm{~cm} \mathrm{H} 2 \mathrm{O} / 40 \mathrm{~s})$. After this maneuver, her positive end-expiratory pressure (PEEP) was maintained at $8 \mathrm{cmH} 20$. At the end of this recruitment procedure, her blood pressure decreased to $86 / 51 \mathrm{mmHg}$, requiring filling with $500 \mathrm{ml}$ crystalloid and two boluses of ephedrine (60mg) to achieve a blood pressure of $112 / 65 \mathrm{mmHg}$. After respiratory and hemodynamic stabilization, maintenance of anesthesia was provided by a mixture of oxygen, nitrous oxide $(60 \%: 40 \%)$ and $2 \%$ of sevoflurane. Airway pressures (Paw) remained at $35 \mathrm{~cm} \mathrm{H2O}$ with a saturation level of $99 \%$ under a fraction of inspired oxygen at $60 \%$ and PEEP of $8 \mathrm{cmH} 20$. Maintenance of the slightly proclive position and opening of the abdomen with the progressive removal of $3200 \mathrm{ml}$ ascitic fluid allowed a lower thoracic pressure $(\mathrm{Paw}=24 \mathrm{~cm} \mathrm{H} 2 \mathrm{O}$ ). Her hemodynamic status remained stable with a filling of $1000 \mathrm{ml}$ of saline serum $(0.9 \%)$. Intraoperative bleeding was estimated at $600 \mathrm{ml}$, her hemoglobin at the end of surgery was $10.9 \mathrm{~g} / \mathrm{dl}$ and no operative transfusion was administered. The surgical procedure consisted of resection of the tumor, hysterectomy, and omentectomy (Figure 2). Her bladder and bowel loops were compressed without evidence of invasion. The surgical intervention required 3 hours and 30 minutes. Diuresis at the end of the surgery was to $550 \mathrm{ml}$. The intraoperative analgesia was provided by paracetamol (1g), nefopam (20mg) and morphine (5mg) by slow infusion. This analgesia was relayed postoperatively by paracetamol $1 \mathrm{~g} / 6 \mathrm{~h}$, nefopam $20 \mathrm{mg} / 8 \mathrm{~h}$, and morphine patient-controlled analgesia (PCA). In the recovery room, extubation was made after warming, wakening and stabilizing of her respiratory and hemodynamic parameters. The monitor showed a blood pressure of $145 / 68 \mathrm{mmHg}$ and blood oxygen saturation (SpO2) of $98 \%$. Our patient remained on oxygen for 2 hours under $4 \mathrm{~L} / \mathrm{min}$ of flow. Postoperative chest radiography showed no worsening of the effusion. Her hemoglobin and postoperative blood electrolytes were unremarkable. Prevention of thromboembolic disease was started $6 \mathrm{~h}$ after the end of the surgery based on enoxaparine $40 \mathrm{mg} / 24 \mathrm{~h}$ and compression stockings. The nature of the tumor histology was thecoma with a benign prognosis, confirming Demons-Meigs' syndrome. Clinical evolution after five months was marked by a complete recovery of our patient and no recurrence of effusion.

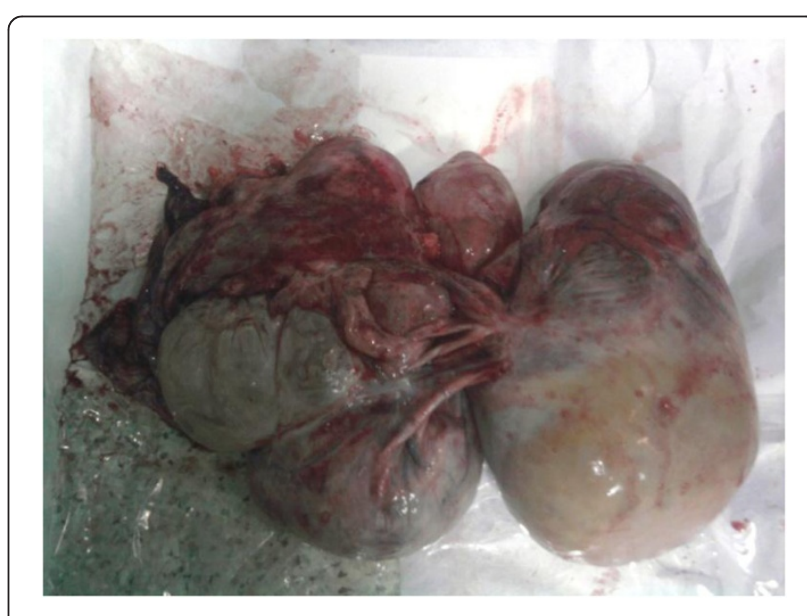

Figure 2 Abdominal tumor encapsulated after resection surgery. 


\section{Discussion}

Demons-Meigs' syndrome can cause severe respiratory and hemodynamic complications during anesthesia caused by a giant mass in the peritoneal space, massive ascites and pleural effusion. Theories to explain the pathophysiology of the peritoneal and pleural effusion have been proposed. Mechanical compression or partial torsion of fibromas or other benign ovarian solid tumors leads to the ascites production by the lymph released from the surface of the tumor [9]. There is exudation from the peritoneum because of mechanical irritation by the heavy mobile tumor [2]. There is injury or necrosis of cyst formations within the tumor [10]. Recently, a probable active secretion by the tumor of the growth factors mediating the hyperpermeability in the ovary or the peritoneal vessel was suggested [11]. In the mechanism of hydrothorax production, ascites are believed to migrate to the right thoracic cavity through a congenital defect or overloaded lymphatics, which are more common on the right [12]. Hence, there is a high incidence of right-sided hydrothorax.

Patients with Demons-Meigs' syndrome complain of abdominal and respiratory symptoms [6]. The increased intraperitoneal pressure, caused by the contents in the peritoneal cavity, can have adverse physiologic effects, such as decreased cardiac output, ventilation-perfusion abnormalities with an accompanying hypoxia and hypercapnia, reduction in renal perfusion flow and glomerular filtration with decreased urine output, elevation in intracranial pressure, and impaired liver and gastrointestinal perfusion with digestive transit disorders. But the true challenge for anesthetists in this syndrome is the abdominal hypertension and its potential evolution to abdominal compartment syndrome $[8,13-15]$. The intra-abdominal hypertension is defined by a sustained or repeated pathological elevation in intra-abdominal pressure $\geq 12 \mathrm{mmHg}$. Evolution can be to abdominal compartment syndrome, which is defined as a sustained intra-abdominal pressure $>20 \mathrm{mmHg}$ that is associated with new organ dysfunction/failure [16].

Different cases of abdominal compartment syndrome complicating Demons-Meigs' syndrome have been reported [8,13-15]. Treatment of this syndrome is based on vascular filling, sedation, analgesia, the reverse Trendelenberg position, neuromuscular blockade and surgical decompression [16]. The indications for this decompression remain controversial. In Demons-Meigs' syndrome, complicated by intra-abdominal hypertension, surgical indication is retained for excision of the abdominal mass even in the absence of intra-abdominal hypertension [8]. In preoperative preparation, measurement of intra-abdominal pressure is made especially to graduate and monitor intra-abdominal hypertension. In the postoperative period, this measurement is necessary in case of massive filling or bleeding with the establishment of packing. In our patient, a measurement of intra-abdominal pressure was not made. Surgical indication was retained for excision of the tumor.

Many patients with Meigs' syndrome are undernourished, with an associated anemia and electrolyte imbalance, which require correction [6]. To overcome these problems, anesthetic management starts with a good preoperative evaluation that assesses the importance of the respiratory, hemodynamic and metabolic impact. Pleural effusion may cause chest pain, shortness of breath; the quantity of liquid is evaluated by a chest X-ray. Extreme, hypoxic respiratory distress requires aspiration of the pleural and abdominal transudates to restore respiratory function and physiology to as near normal limits as possible. Aspiration should be repeated if necessary: the final aspirates being done on the day before the operation [6].

In our patient, several factors contributed to the desaturation, the abdominal compartment syndrome related to Demons-Meigs' syndrome and the formation of atelectasis, related to anesthetic induction and especially paralysis. To correct these intraoperative desaturation problems, several procedures were used, however, alveolar recruitment maneuvers remained the most effective procedure [17-19]. The only limitation of these maneuvers is their hemodynamic consequences. In our case desaturation was deep, authorizing the use of these maneuvers. The decrease in blood pressure was corrected by volume expansion and vasoconstrictors.

Preoperative anemia is common Demons-Meigs' syndrome [6] and is associated with increased likelihood of blood transfusion and increased perioperative morbidity. Correction by transfusion of red blood cells or whole blood, when they are necessary, is required. Other electrolytic disorders should be corrected before surgery. Premedication with hydroxizine has been shown to be safe. An opioid-based or benzodiazepine medication should be avoided because of the risk of preoperative respiratory depression, hypoventilation and hypoxemia [6]. Because of risk of regurgitation consequent of the increase in the intra-abdominal pressure and restricted diaphragmatic movement, the anesthetic technique is a crash induction with application of the Sellick's maneuver after preoxygenation in a proclive position. Thoracic pressures are generally high, this problem can be solved by proclive positioning, rapid laparotomy to drain the ascites, and incidental use of autoflow ventilator mode. A postoperative chest X-ray to search for residual pleural effusion is indicated. It is rarely necessary to perform chest aspiration following the operation, but hypoxia during recovery has been described [3].

Excision of a huge tumor is associated with risk of bleeding due to the size of the tumor, and its anatomical relationships. It can result in hemodynamic intraoperative alteration, aggravated by the inconvenience of venous return due to abdominal pressure. This requires adequate 
intraoperative monitoring, performing of transfusion laboratory tests, anticipation of blood loss by optimizing blood volume and transfusion thresholds accordingly. As in any oncologic pelvic surgery, antibiotic prophylaxis is based on a first- or second-generation cephalosporin.

The management of postoperative pain is made by a multimodal analgesia and intravenous opioids. The use of epidural analgesia has been described as an efficient option in such cases [7]. Prevention of thromboembolic events is achieved by low-molecular-weight heparin combined with compression stockings and early ambulation.

\section{Conclusions}

Demon-Meigs' syndrome is a benign disease with a good prognosis. The respiratory and hemodynamic risks of this syndrome are the main anesthetic problems. Perioperative optimal management of these risks preserves the good prognosis of this syndrome.

\section{Consent}

Written informed consent was obtained from the patient for publication of this manuscript and any accompanying images. A copy of the written consent is available for review by the Editor-in-Chief of this journal.

\section{Abbreviations}

BMI: body mass index; CA: carbohydrate antigen; CT: computed tomography; NYHA: New York Heart Association; Paw: airway pressures; PCA: patient-controlled analgesia; PEEP: positive end-expiratory pressure; SpO2: blood oxygen saturation.

\section{Competing interests}

The authors declare that they have no competing interests.

\section{Authors' contributions}

$\mathrm{SF}$ and $\mathrm{MB}$ analyzed and interpreted the patient data. SF and MB were major contributors in writing the manuscript. $\mathrm{CH}$ and $\mathrm{HA}$ made the final corrections. All authors read and approved the final manuscript.

Received: 8 April 2014 Accepted: 17 June 2014

Published: 27 September 2014

\section{References}

1. Peparini N, Chirletti P: Ovarian malignancies with cytologically negative pleural and peritoneal effusions: demons' or Meigs' pseudo-syndromes? Int J Surg Pathol 2009, 17:396-397.

2. Meigs JV: Fibroma of the ovary with ascites and hydrothorax: Meigs' syndrome. Am J Obstet Gynecol 1954, 67:962-985.

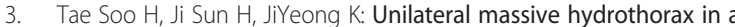
gynecologic patient with pseudo-Meigs' syndrome. A case report. Korean J Anesthesiol 2010, 58:202-206.

4. Maiga B, Dolo A, Dembele M, Traore HA, Leroy P, Pichard E: Meigs syndrome. About one case. Gynecol Obstet Fertil 2003, 31:240-242.

5. Bhatla N: Jeffcoate's Principles of Gynecology. International Edn. Revised, and Updated from the 5th Edn. London: Arnold publishers; 2001:523-524.

6. Patrick B, Donnelly MB: Anaesthesia in Meig's syndrome. Anaesthesia 1966, 21:2.

7. Hirota M, Noda J, Katoh S, Hotta R, Furuhashi Y, Suzuki S: Perioperative management of patients with Meigs syndrome. Masui 1995, 44:874-879.

8. Peparini N, Di Matteo FM, Silvestri A, Caronna R, Chirletti P: Abdominal hypertension in Meigs' syndrome. Eur J Surg Oncol 2008, 34:938-942.

9. Samanth KK, Black WC III: Benign ovarian stromal tumors associated with free peritoneal fluid. Am J Obstet Gynecol 1970, 107:538-545.
10. Abad A, Cazorla E, Ruiz F, Aznar I, Asins E, Llixiona J: Meigs' syndrome with elevated CA125: case report and review of the literature. Eur J Obstet Gynecol Reprod Biol 1999, 82:97-99.

11. Abramov Y, Anteby SO, Fasouliotis SJ, Barak V: Markedly elevated levels of vascular endothelial growth factor, fibroblast growth factor, and interleukin 6 in Meigs' syndrome. Am J Obstet Gynecol 2001, 184:354-355.

12. Datta R, Sharma P, Choudhury S, Sarkar S: Meigs syndrome - a case report. J Obstet Gynecol India 2006, 56:451-453.

13. Merlicco D, Roggia G, Lombardi M, Lattanzio L, Marzaioli R, Nacchiero M: Abdominal compartment syndrome due to a giant multilobulated ovarian serous cystadenoma. Case report and review of the literature. Ann Ital Chir 2012, 83:563-566.

14. Sabri N1, Athavale R: Abdominal compartment syndrome: a rare complication of an ovarian tumour. J Obstet Gynaecol 2013, 33:744-745.

15. Giuliani A, Basso L, Demoro M, Scimo' M, Galati F, Galati G: Bilateral ovarian mucinous cystadenoma in an adolescent presenting as abdominal compartment syndrome. Eur J Obstet Gynecol Reprod Biol 2008, 140:278-279.

16. Kirkpatrick AW, Roberts DJ, De Waele J, Jaeschke R, Malbrain ML, De Keulenaer B, Duchesne J, Bjorck M, Leppaniemi A, Ejike JC, Sugrue M, Cheatham M, Ivatury R, Ball CG, Reintam Blaser A, Regli A, Balogh Z, D'Amours S, Debergh D, Kaplan M, Kimball E, Olvera C, Pediatric Guidelines Sub-Committee for the World Society of the Abdominal Compartment Syndrome: Intra-abdominal hypertension and the abdominal compartment syndrome: updated consensus definitions and clinical practice guidelines from the world society of the abdominal compartment syndrome. Intensive Care Med 2013, 39:1190-1206.

17. Aldenkortt M, Lysakowski C, Elia N, Brochard L, Tramèr MR: Ventilation strategies in obese patients undergoing surgery: a quantitative systematic review and meta-analysis. Br J Anaesth 2012, 109:493-502.

18. Futier E, Constantin JM, Pelosi P, Chanques G, Kwiatkoskwi F, Jaber S, Bazin JE: Intraoperative recruitment maneuver reverses detrimental pneumoperitoneum-induced respiratory effects in healthy weight and obese patients undergoing laparoscopy. Anesthesiology 2010, 113:1310-1319.

19. Reinius H, Jonsson L, Gustafsson S, Sundbom M, Duvernoy O, Pelosi P, Hedenstierna G, Fredén F: Prevention of atelectasis in morbidly obese patients during general anesthesia and paralysis: a computerized tomography study. Anesthesiology 2009, 111:979-987.

doi:10.1186/1752-1947-8-320

Cite this article as: Fjouji et al:: Anesthetic considerations in Demons-Meigs' syndrome: a case report. Journal of Medical Case Reports 2014 8:320.

\section{Submit your next manuscript to BioMed Central and take full advantage of:}

- Convenient online submission

- Thorough peer review

- No space constraints or color figure charges

- Immediate publication on acceptance

- Inclusion in PubMed, CAS, Scopus and Google Scholar

- Research which is freely available for redistribution 